\title{
"Beat" patterns for the odd-even staggering in octupole bands from a quadrupole--octupole Hamiltonian
}

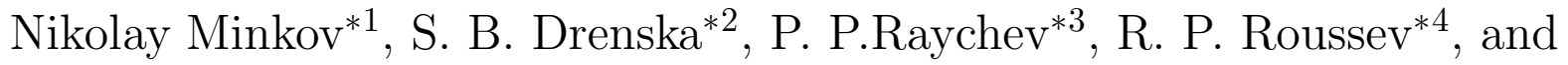 \\ Dennis Bonatsos抑 \\ * Institute for Nuclear Research and Nuclear Energy, \\ 72 Tzarigrad Road, 1784 Sofia, Bulgaria \\ † Institute of Nuclear Physics, N.C.S.R. "Demokritos", \\ GR-15310 Aghia Paraskevi, Attiki, Greece
}

\begin{abstract}
We propose a collective Hamiltonian which incorporates the standard quadrupole terms, octupole terms classified according to the irreducible representations of the octahedron group, a quadrupole-octupole interaction, as well as a term for the bandhead energy linear in $K$ (the projection of angular momentum on the body-fixed $z$-axis). The energy is subsequently minimized with respect to $K$ for each given value of the angular momentum $I$, resulting in $K$ values increasing with $I$ within each band, even in the case in which $K$ is restricted to a set of microscopically plausible values. We demonstrate that this Hamiltonian is able to reproduce a variety of "beat" patterns observed recently for the odd-even staggering in octupole bands of light actinides.
\end{abstract}

PACS Numbers: 21.60.Fw, 21.60.Ev, 21.10.Re

\footnotetext{
${ }^{1}$ e-mail: nminkov@inrne.bas.bg

${ }^{2}$ e-mail: sdren@inrne.bas.bg

${ }^{3}$ e-mail: raychev@inrne.bas.bg

${ }^{4}$ Deceased

${ }^{5}$ e-mail: bonat@mail.demokritos.gr
} 


\section{Introduction}

The properties of nuclear systems with octupole deformations [1] are of current interest due to increasing evidence for the presence of octupole instabilities in various regions of the nuclear table [2, 3, 4]. Furthermore, some "beat" patterns have been observed recently for the odd-even staggering (the relative displacement of the odd levels with respect to the positions at which they should have been located according to a fit of the even levels by the formula $E(I)=A I(I+1)$, where $I$ denotes the angular momentum) in octupole bands of light actinides [5] based on recent experimental data [6, 7], calling for a study of the interactions which could give rise to such shapes.

Various parametrizations of the octupole degrees of freedom [8, 9, 10, 11] already exist, being a useful tool for understanding the role of the reflection asymmetry correlations and for analyzing the collective properties of such systems. Some important questions in this direction are: which are the collective nuclear interactions that correspond to the different octupole shapes and how do they determine the structure of the respective energy spectra? Physically meaningful answers should be obtained by taking into account the simultaneous presence of other collective degrees of freedom, such as the quadrupole ones [12].

In the present work we address the above problems by examining the interactions that generate collective rotations in a system with a simultaneous presence of octupole and quadrupole deformations. The basic assumption of our consideration is that the rotational motion of such a system can be interpreted in first approximation as the motion of a body with a stable quadrupole-octupole shape. In this respect our purpose is to examine how the nuclear system behaves under collective rotations if the presence of stable quadrupoleoctupole deformations is assumed.

Based on the octahedron point symmetry parametrization of the octupole shape [8, 9, 10, we propose a collective Hamiltonian which incorporates the interactions responsible for the rotations associated with the different octupole deformations. In addition we take into account the quadrupole degrees of freedom and the appropriate higher order quadrupoleoctupole interaction. Below it will be shown that such a general model Hamiltonian could incorporate the basic properties of a nuclear system rotating under the above assumption.

Although this assumption seems to be rather strong (since the presence of stable octupole deformations in nuclei is not yet a well elucidated problem) we suppose that it could give a natural possibility to estimate the extent to which some of the observed nuclear octupole bands carry the characteristics of the stable octupole shapes. Generally, the proposed consideration will provide a direct physical insight into the nuclear collective motion as far as the shape of the system and the respective moments of inertia are slightly changed under the collective motion. Similarly to the case of the pure quadrupole deformations this requirement will be naturally satisfied for the low angular momentum region of the spectrum which is, from another perspective, accessible for detailed microscopic analysis, the length of this region depending on the particular system.

Furthermore, we expect that in the higher angular momentum regions the approach suggested will outline some general properties of the system and thus will provide a relevant guide for respective more detailed studies from both microscopic and phenomenological points of view. In particular it will be shown that the model formalism developed in the present work proposes a schematic explanation of the recently observed [5] "beat" 
patterns for the odd-even staggering in octupole bands of light actinides based on recent experimental data [6, 7].

In Section 2 of the present work the octupole terms of the Hamiltonian, classified by the irreducible representations (irreps) of the octahedron group, will be described, while the quadrupole terms and the octupole-quadrupole interaction will be examined in Section 3, along with the bandhead term of the Hamiltonian and the minimization procedure, which is a basic ingredient of the present work. In Section 4 the diagonal parts of the Hamiltonian will be analyzed and used for the production of schematic odd-even staggering patterns, while the same procedure will be repeated including the non-diagonal parts of the Hamiltonian in Section 5. In Section 6 an analysis of the model formalism under some restrictions on the permitted values of the angular momentum projection on the body-fixed $z$-axis will be presented, while further tests of the formalism will be performed in Section 7. Finally, Section 8 will contain discussion of the present results, while in Section 9 a summary of the present results and plans for future work will be given.

\section{Parametrization of the octupole deformation}

Our model formalism is based on the principle that the collective properties of a physical system in which octupole correlations take place can be expressed by the following most general octupole field in the intrinsic (body-fixed) frame 9]

$$
V_{3}=\sum_{\mu=-3}^{3} \alpha_{3 \mu}^{f i x} Y_{3 \mu}^{*}
$$

with $\left(\alpha_{3 \mu}^{f i x}\right)^{*}=(-1)^{\mu} \alpha_{3-\mu}^{f i x}$

This field can be written in the form [9]

$$
V_{3}=\epsilon_{0} A_{2}+\sum_{i=1}^{3} \epsilon_{1}(i) F_{1}(i)+\sum_{i=1}^{3} \epsilon_{2}(i) F_{2}(i),
$$

where the quantities 9

$$
\begin{aligned}
A_{2} & =-\frac{i}{\sqrt{2}}\left(Y_{32}-Y_{3-2}\right)=\frac{1}{r^{3}} \sqrt{\frac{105}{4 \pi}} x y z \\
F_{1}(1) & =Y_{30}=\frac{1}{r^{3}} \sqrt{\frac{7}{4 \pi}} z\left(z^{2}-\frac{3}{2} x^{2}-\frac{3}{2} y^{2}\right) \\
F_{1}(2) & =-\frac{1}{4} \sqrt{5}\left(Y_{33}-Y_{3-3}\right)+\frac{1}{4} \sqrt{3}\left(Y_{31}-Y_{3-1}\right) \\
& =\frac{1}{r^{3}} \sqrt{\frac{7}{4 \pi}} x\left(x^{2}-\frac{3}{2} y^{2}-\frac{3}{2} z^{2}\right), \\
F_{1}(3) & =-i \frac{1}{4} \sqrt{5}\left(Y_{33}+Y_{3-3}\right)-i \frac{1}{4} \sqrt{3}\left(Y_{31}+Y_{3-1}\right) \\
& =\frac{1}{r^{3}} \sqrt{\frac{7}{4 \pi}} y\left(y^{2}-\frac{3}{2} z^{2}-\frac{3}{2} x^{2}\right)
\end{aligned}
$$




$$
\begin{aligned}
F_{2}(1) & =\frac{1}{\sqrt{2}}\left(Y_{32}+Y_{3-2}\right)=\frac{1}{r^{3}} \sqrt{\frac{105}{16 \pi}} z\left(x^{2}-y^{2}\right) \\
F_{2}(2) & =\frac{1}{4} \sqrt{3}\left(Y_{33}-Y_{3-3}\right)+\frac{1}{4} \sqrt{5}\left(Y_{31}-Y_{3-1}\right) \\
& =\frac{1}{r^{3}} \sqrt{\frac{105}{16 \pi}} x\left(y^{2}-z^{2}\right) \\
F_{2}(3) & =-i \frac{1}{4} \sqrt{3}\left(Y_{33}+Y_{3-3}\right)+i \frac{1}{4} \sqrt{5}\left(Y_{31}+Y_{3-1}\right) \\
& =\frac{1}{r^{3}} \sqrt{\frac{105}{16 \pi}} y\left(z^{2}-x^{2}\right)
\end{aligned}
$$

(with $r^{2}=x^{2}+y^{2}+z^{2}$ ) belong to irreducible representations (irreps) of the octahedron group $O$. In particular, the first quantity (Eq. (3) ) belongs to the one-dimensional irrep $A_{2}$, while the next three quantities (Eqs (4)-(6)) belong to the three-dimensional irrep $F_{1}$ and the last three quantities (Eqs (7)-(9) ) belong to the three-dimensional irrep $F_{2}$. The seven real parameters $\epsilon_{0}$ and $\epsilon_{r}(i)(r=1,2 ; i=1,2,3)$, appearing in Eq. (2), determine the amplitudes of the various components of the octupole deformation. Their relation to the coefficients $\alpha_{3 \mu}^{f i x}$ has been given in Ref. [9].

As we have already seen, the quantities appearing in Eqs (3)-(9), when expressed in terms of the cartesian coordinates $x, y$, and $z$, contain linear combinations of terms cubic in the cartesian variables. These specific linear combinations correspond to various octupole shapes (as seen from their expressions in terms of the spherical harmonics), and in addition correspond to the above mentioned irreps of the octahedron group. Our proposition is the following:

a) We construct a Hamiltonian using the same cubic terms appearing in Eqs (3)-(9), but replacing the cartesian coordinates $x, y, z$ by the angular momentum operators $\hat{I}_{x}, \hat{I}_{y}$, $\hat{I}_{z}\left(\right.$ with $\hat{I}^{2}=\hat{I}_{x}^{2}+\hat{I}_{y}^{2}+\hat{I}_{z}^{2}$ ). For example, the term $z^{3}$ is replaced by $\hat{I}_{z}^{3}$.

b) Due to the fact that the operators $\hat{I}_{x}, \hat{I}_{y}, \hat{I}_{z}$ do not commute, while the coordinates $x, y, z$ do commute, when making step a) we symmetrize each cubic expression when we write it in terms of the angular momentum operators. For example, the term $z\left(x^{2}-y^{2}\right)$ is replaced by $\hat{I}_{z}\left(\hat{I}_{x}^{2}-\hat{I}_{y}^{2}\right)+\left(\hat{I}_{x}^{2}-\hat{I}_{y}^{2}\right) I_{z}$.

c) During the procedure described above, the $r^{3}$ factors appearing in the denominators of Eqs (3)-(9) are replaced by $\hat{I}^{3}$ factors. In the final result we normalize with respect to $\hat{I}^{3}$, i.e. we multiply the results by $\hat{I}^{3}$, an operation which is equivalent to the transition to a unit sphere, a natural thing to do since we are interested in surface shapes. Indeed, this operation is equivalent to the multiplication of the quantities appearing in Eqs (3)-(9) by $r^{3}$, an action which eliminates their radial dependence, leading to a transition to the unit sphere. The transition to the unit sphere is the reason that the standard quadrupole and octupole operators are defined to be proportional to $r^{2} Y_{2 \mu}$ and $r^{3} Y_{3 \mu}$ respectively [1].

d) As a result, we obtain a Hamiltonian (as a function of the angular momentum operators $\hat{I}_{x}, \hat{I}_{y}, \hat{I}_{z}$ ) the terms of which correspond to the same octupole shapes which appear in the Hamiltonian of Eqs (2)-(9). 
e) The terms of the resulting Hamiltonian in addition belong to the same irreps of the octahedron group as the terms of the original Hamiltonian, appearing in Eqs (3)(9). In other words, through this procedure we determine the octahedron point symmetry properties of the system in angular momentum space.

Our proposition is similar to the procedure used in Ref. [13 for the hexadecapole field.

The following Hamiltonian is then obtained:

$$
\hat{H}_{o c t}=\hat{H}_{A_{2}}+\sum_{r=1}^{2} \sum_{i=1}^{3} \hat{H}_{F_{r}(i)}
$$

with

$$
\begin{aligned}
\hat{H}_{A_{2}} & =a_{2} \frac{1}{4}\left[\left(\hat{I}_{x} \hat{I}_{y}+\hat{I}_{y} \hat{I}_{x}\right) \hat{I}_{z}+\hat{I}_{z}\left(\hat{I}_{x} \hat{I}_{y}+\hat{I}_{y} \hat{I}_{x}\right)\right] \\
\hat{H}_{F_{1}(1)} & =\frac{1}{2} f_{11} \hat{I}_{z}\left(5 \hat{I}_{z}^{2}-3 \hat{I}^{2}\right) \\
\hat{H}_{F_{1}(2)} & =\frac{1}{2} f_{12}\left(5 \hat{I}_{x}^{3}-3 \hat{I}_{x} \hat{I}^{2}\right) \\
\hat{H}_{F_{1}(3)} & =\frac{1}{2} f_{13}\left(5 \hat{I}_{y}^{3}-3 \hat{I}_{y} \hat{I}^{2}\right) \\
\hat{H}_{F_{2}(1)} & =f_{21} \frac{1}{2}\left[\hat{I}_{z}\left(\hat{I}_{x}^{2}-\hat{I}_{y}^{2}\right)+\left(\hat{I}_{x}^{2}-\hat{I}_{y}^{2}\right) \hat{I}_{z}\right] \\
\hat{H}_{F_{2}(2)} & =f_{22}\left(\hat{I}_{x} \hat{I}^{2}-\hat{I}_{x}^{3}-\hat{I}_{x} \hat{I}_{z}^{2}-\hat{I}_{z}^{2} \hat{I}_{x}\right) \\
\hat{H}_{F_{2}(3)} & =f_{23}\left(\hat{I}_{y} \hat{I}_{z}^{2}+\hat{I}_{z}^{2} \hat{I}_{y}+\hat{I}_{y}^{3}-\hat{I}_{y} \hat{I}^{2}\right)
\end{aligned}
$$

The Hamiltonian parameters $a_{2}$ and $f_{r i}(r=1,2 ; i=1,2,3)$, appearing in Eqs (11)-(17), are formally related to the parameters in Eq. (2) as follows

$$
a_{2}=\epsilon_{0} \sqrt{\frac{105}{4 \pi}}, \quad f_{1 i}=\epsilon_{1}(i) \sqrt{\frac{7}{4 \pi}}, \quad f_{2 i}=\epsilon_{2}(i) \sqrt{\frac{105}{16 \pi}}, \quad i=1,2,3 .
$$

The non-vanishing matrix elements of the operators of Eqs (11)-(17) in the states with collective angular momentum $I$ are given in the Appendix.

In the Appendix we remark that the operator $\hat{H}_{F_{1}(1)}$ (Eq. (12)), which corresponds to the term $Y_{30}$ (see Eq. (4) ), characterized by axial deformation, is the only octupole operator possessing diagonal matrix elements. Below it will be seen that this operator is of major importance in determining the fine structure of collective bands with octupole correlations. This fact does not come as a surprise, since it is well known that the $Y_{30}$ (axial) deformation is the leading mode in systems with reflection asymmetric shapes (see Ref. [4 for a relevant review).

\section{Inclusion of quadrupole degrees of freedom}

It is known, however, that the use of the pure octupole field of Eq. (11) is not sufficient for the description of the collective properties of nuclei exhibiting octupole deformation, since 
the quadrupole deformation is also present. Therefore one has to consider the octupole degrees of freedom together with the quadrupole deformations, and in addition one has to deal with their coupling. A general treatment of a combined quadrupole-octupole field has been given earlier in the framework of a general collective model for coupled multipole surface modes [2, [12].

According to the above considerations it is reasonable to suggest that the most general collective Hamiltonian of a system exhibiting octupole deformations should also contain the standard (axial) quadrupole rotation part

$$
\hat{H}_{\text {rot }}=A \hat{I}^{2}+A^{\prime} \hat{I}_{z}^{2}
$$

where $A$ and $A^{\prime}$ are the inertial parameters. In addition, it is reasonable to try to describe the coupling between the quadrupole and the octupole degrees of freedom by the following higher order diagonal quadrupole-octupole interaction term, which corresponds to the product $Y_{20} Y_{30}$,

$$
\hat{H}_{q o c}=f_{q o c} \frac{1}{I^{2}}\left(15 \hat{I}_{z}^{5}-14 \hat{I}_{z}^{3} \hat{I}^{2}+3 \hat{I}_{z} \hat{I}^{4}\right),
$$

since it is known that the axial deformation corresponding to $Y_{30}$ is the leading mode in systems with reflection asymmetric shapes [4] and, in an analogous way, the axial deformation corresponding to $Y_{20}$ is the leading mode in systems with quadrupole deformations. The operator of Eq. (20) is also normalized with respect to the multiplication factor $I^{3}$, in the same way as the operators of Eqs (11)-(17) are, as described in the previous Section. In other words, we use the product $I^{3} Y_{20} Y_{30}$, in order to ensure that all non-quadrupole Hamiltonian terms will be of the same order.

As a result, the total Hamiltonian of the system can be written in the form

$$
\hat{H}=\hat{H}_{b h}+\hat{H}_{\text {rot }}+\hat{H}_{o c t}+\hat{H}_{q o c} \text {, }
$$

where

$$
\hat{H}_{b h}=\hat{H}_{0}+f_{k} \hat{I}_{z}
$$

is a pure phenomenological part introduced in order to reproduce the bandhead energy in the form

$$
E_{b h}=E_{0}+f_{k} K
$$

were $E_{0}$ and $f_{k}$ are free parameters. The bandhead energy $E_{b h}$ is considered proportional to $K$ in analogy to what happens in the five-dimensional quadrupole oscillator model (see Appendix 6B of Ref. [回]), as well as in the standard rotation-vibration model (see section 6.5 of Ref. [14). The $K$-dependence of $E_{b h}$ plays an important role in our approach, since it provides the correct value of the bandhead angular momentum projection $K$ in the variation procedure described below.

We remark that the Hamiltonian of Eq. (21) is not a rotational invariant in general. It does not commute with the total angular momentum operators and, as a result, any state with given angular momentum $I$ is energy split with respect to the quantum number $K$. Therefore, the physical relevance of this Hamiltonian depends on the possibility to determine in a unique way the angular momentum projection. Our basic assumption is that $K$ is not frozen within the sequence of states of the collective rotational band. We suggest that for any given angular momentum $I$ the projection $K$ should be determined so as to minimize the respective collective energy. The resulting octupole band is then the yrast sequence of the energy levels produced by our model Hamiltonian. It should be mentioned that a similar procedure has been used in Refs [15, 16] in relation to the $\Delta I=2$ staggering effect in superdeformed nuclei. 


\section{The diagonal parts of the Hamiltonian}

As a first step in testing our Hamiltonian we consider its diagonal part

$$
\hat{H}^{d}=\hat{H}_{b h}+\hat{H}_{r o t}+\hat{H}_{o c t}^{d}+\hat{H}_{q o c}
$$

where the operator $\hat{H}_{o c t}^{d} \equiv \hat{H}_{F_{1}(1)}$ represents the diagonal part of the pure octupole Hamiltonian $\hat{H}_{\text {oct }}$ of Eq. (10).

The following diagonal matrix element is then obtained

$$
\begin{aligned}
E_{K}(I) & =E_{0}+f_{k} K+A I(I+1)+A^{\prime} K^{2}+f_{11}\left(\frac{5}{2} K^{3}-\frac{3}{2} K I(I+1)\right) \\
& +f_{q o c} \frac{1}{I^{2}}\left(15 K^{5}-14 K^{3} I(I+1)+3 K I^{2}(I+1)^{2}\right) .
\end{aligned}
$$

Following the above mentioned assumption for the angular momentum projection $K$, we determine the yrast sequence $E(I)$ by minimizing for each value of $I$ the expression of Eq. (25) as a function of integer $K$ in the range $-I \leq K \leq I$. The obtained energy spectrum depends on six model parameters: $E_{0}$ is a constant contributing to the bandhead energy; $f_{k}$ is determined in order to correspond to the correct bandhead energy of the octupole band for $I=1$, in which case one has $K=1$ as well (This is appropriately modified in the case of bands starting with higher $K$. For example in $K=5$ bands the lowest angular momentum is $I=5$, and $f_{k}$ is fixed in order to provide the correct bandhead energy for $I=K=5$.); $A$ and $A^{\prime}$ are the quadrupole inertial parameters which should in general correspond to the known quadrupole shape (axes ratio) of the relevant nucleus; $f_{11}$ and $f_{q o c}$ are the parameters of the diagonal octupole (Eq. (12) ) and quadrupole-octupole (Eq. (20) ) interactions respectively. In what follows we give fixed values to the first four parameters $\left(E_{0}, f_{k}, A, A^{\prime}\right)$ and vary the last two parameters $\left(f_{11}, f_{q o c}\right)$ in order to examine the influence of the last two terms of Eq. (25) on the odd-even staggering pattern.

A schematic energy spectrum of this kind, obtained for a sample set of parameter values, is given in Table 1. It is seen that the "yrast" values of the quantum number $K$, resulting from the minimization procedure described above, gradually increase with the increase of the angular momentum $I$. We remark that these values of $K$ correspond to the local minima of the energy expression of Eq. (25) as a function of $K$, which is illustrated in Fig. 1 for the same set of parameters. We see that these minima appear for positive values of $K$, are well determined, and their depth increases with the increase of the angular momentum $I$.

These results indicate that the rotational motion of a system with stable quadropoleoctupole deformation is associated with complex angular momentum dynamics, due to the complex shape contributions to collectivity. More precisely, the following theoretical proposition is formed: For this kind of rotational motion the increase in the total angular momentum of the system is associated with an energetically favorable increase in its third projection $K$ on the body fixed axis. This means that the vector of the angular momentum deviates "step by step" from the " $x-y$ " body-fixed plane (which is perpendicular to the bodyfixed " $\mathrm{z}$ "-axis) as its magnitude increases. In other words, the higher angular momenta will be attended by stronger precession, or "wobbling motion" of the system. These findings resemble the recent results [17] obtained within the framework of a self-consistent cranked 
HFB approach. Application of the method to some Os isotopes has demonstrated that high- $K$ bands become important in the high angular momentum region [17].

Such a behavior of the spectrum could also be interpreted as a multiband-crossing phenomenon, since the obtained yrast sequence can be considered as the envelope of the curves with different values of the quantum number $K$, as it is illustrated in Fig. 2. This interpretation can, however, be considered as a mathematical description, not necessarily implying the physical existence of several bands with different values of $K$.

Thus, our schematic consideration suggests that the rotational motion of a system with stable quadrupole-octupole deformations invokes $K$-values essentially higher than the ones usually considered in microscopic studies. We remark that the situation considered here is essentially different from the case of pure quadrupole rotations, where the bandhead $K$-value always provides the minimum of the collective rotational energy along the whole band (for example $\left.E_{\text {rot }}=A I(I+1)+A^{\prime} K^{2}\right)$. Thus the obtained wobbling motion appears as an effect of the higher multipolarity of the considered collective interactions. The extent to which the $K$-values appearing in our procedure provide physically reasonable interpretations of various nuclear collective modes will be considered in Section 6 , where the limitation to $K \leq 3$ will be studied.

Now we can examine the fine structure of the collective bands obtained in the above model procedure. We see in Table 1 that the $K$-values characterizing the levels with increasing $I$ are grouped in couples containing two successive values of $I$ (one even and one odd). This fact implies the presence of an odd-even staggering effect, as we shall immediately see. A measure of the odd-even staggering effect is the quantity

$$
\operatorname{Stg}(I)=6 \Delta E(I)-4 \Delta E(I-1)-4 \Delta E(I+1)+\Delta E(I+2)+\Delta E(I-2),
$$

with

$$
\Delta E(I)=E(I+1)-E(I) .
$$

The quantity $\operatorname{Stg}(I)$ is proportional to the discrete approximation of the fourth derivative of the function $\Delta E(I)$, i.e. proportional to the fifth derivative of the energy $E(I)$, and is able to demonstrate fine deviations from the pure rotational behavior, as it has been demonstrated in Ref. [5] for the octupole bands of several light actinides. An analog of this quantity has been introduced earlier [18, 19, 20 for the study of $\Delta I=2$ staggering in nuclear superdeformed bands.

The way in which the appearance of the $K$-values in couples for successive values of $I$ leads to odd--even staggering can be seen from Table 1, using the second term of Eq. (25) as an example. In Table 1 we see that couples of $K$ values appear for $I \geq 8$. In this region the contribution of the second term of Eq. (25) to $\Delta E(I)$ is zero for even values of $I$, while it is $+f_{k}$ for odd values of $I$. Then we easily see from Eq. (26) that the contribution of the second term of Eq. (25) to $S t g(I)$ is $+8 f_{k}$ for odd values of $I$, while it is $-8 f_{k}$ for even values of $I$. Therefore the second term of Eq. (25) leads to odd-even staggering of constant amplitude. The 4th, 5th and 6th terms of Eq. (25) depend nonlinearly on $K$ and/or $I$, and therefore give odd-even staggering of varying amplitude. In the case of the 4th term, it is easy to see that it gives staggering with amplitude increasing linearly as a function of I. (The terms $E_{0}$ and $A I(I+1)$ do not contribute to the odd-even staggering, as it is known from Ref. [5].) 
Several examples of odd-even staggering occurring from Eq. (26) for different sets of parameter values used in Eq. (25) are given in Fig. 3. The parameters $E_{0}$ and $f_{k}$ have been kept constant in all parts of Fig. 3, while the parameters $A$ and $A^{\prime}$ have been kept constant in all parts of Fig. 3 except the last one (Fig. 3(f) ). In contrast, the parameters $f_{11}$ and $f_{\text {qoc }}$ have been given different values in the various parts of Fig. 3.

Fig. 3(a) illustrates a long odd-even staggering pattern which looks similar to the "beats" observed in the octupole bands of some light actinides [5] (with ${ }^{220} \mathrm{Ra},{ }^{224} \mathrm{Ra}$ and ${ }^{226}$ Ra being probably the best examples), a difference being that in the realistic cases of Ref. [5] the amplitude of the "beats" seems to be decreasing with increasing $I$, while in Fig. 3(a) the opposite seems to hold.

In Fig. 3(b) the increased values of $f_{11}$ and $f_{q o c}$ provide a wide angular momentum region (up to $I \sim 40$ ) with a regular staggering pattern.

The further increase of $f_{\text {qoc }}$ results in a staggering pattern with different amplitudes, shown in Fig. 3(c).

The further increase of $f_{11}$ and $f_{q o c}$ leads to a staggering pattern with many "beats", as shown in Fig 3(d). Notice that in Fig. 3(d) the first three "beats" are completed by $I \approx 40$, while in Fig. 3(a) the first three "beats" are completed by $I \approx 70$.

Fig. 3(e) illustrates what happens in the case of vanishing quadrupole-octupole interaction term $\left(f_{\text {qoc }}=0\right)$, keeping the rest of the parameters the same as in Figs 3(b) and 3(c). We see that in the present case the "beat" effect occurs very frequently, while in Figs 3(b) and 3(c) the change was much slower.

Finally, an example with almost constant staggering amplitude is shown in Fig. 3(f). It resembles the form of the odd-even staggering predicted in the $\mathrm{SU}(3)$ limit of various algebraic models (see Ref. [5] for details and relevant references). It also resembles the odd-even staggering seen in some octupole bands of light actinides [5] (with ${ }^{222} \mathrm{Rn}$ being probably the best example).

\section{The non-diagonal parts of the Hamiltonian}

After seeing in the previous section the main features of the diagonal part of the Hamiltonian, we can now focus our attention on the general Hamiltonian of Eq. (21), including the various non-diagonal terms given in Eqs (11), (13)-(17). The main problem in this case is the fact that $K$ is in general not a good quantum number. We can, however, make analysis for small values of the respective parameters $\left(a_{2}, f_{12}, f_{13}, f_{21}, f_{22}, f_{23}\right)$, which keep $K$ "asymptotically" good. Keeping these parameters small means that we use a weak $K$-bandmixing interaction, which guarantees that for any explicit energy minimum appearing in the diagonal case the corresponding eigenvalue of the perturbed Hamiltonian will be uniquely determined. Thus we are able to obtain for the perturbed Hamiltonian a $K$-mixed yrast energy sequence analogous to the one we got in the previous section for the diagonal part of the Hamiltonian.

Our numerical analysis of the Hamiltonian eigenvector systems shows that the parameters of the non-diagonal terms should be smaller by an order of magnitude in comparison to the value of the parameter $f_{11}$. In addition, by examining the corresponding matrix elements in the Appendix, we deduce that the following pairs of non-diagonal terms give 
identical contributions to the energy spectrum: $\hat{H}_{A_{2}}$ and $\hat{H}_{F_{2}(1)} ; \hat{H}_{F_{1}(2)}$ and $\hat{H}_{F_{1}(3)} ; \hat{H}_{F_{2}(2)}$ and $\hat{H}_{F_{2}(3)}$. Therefore it suffices to keep from now on only the terms $\hat{H}_{F_{1}(2)}, \hat{H}_{F_{2}(1)}, \hat{H}_{F_{2}(2)}$.

In Fig. 4 two staggering patterns in the presence of $K$-bandmixing terms are illustrated. In the calculation we have included, as we have already mentioned, the non-diagonal terms $\hat{H}_{F_{1}(2)}, \hat{H}_{F_{2}(1)}$, and $\hat{H}_{F_{2}(2)}$, along with the already considered diagonal Hamiltonian of Eq. (24). The three non-diagonal terms have been included with two different sets of parameters (both obeying the above mentioned condition that the values of the parameters accompanying the non-diagonal terms should be at least an order of magnitude smaller than the value of the parameter $f_{11}$ ), shown in Figs $4(\mathrm{a})$ and $4(\mathrm{~b})$, while the parameters of the diagonal part have been kept the same as in Fig. 3(b) (and in Table 1). Comparing Figs 3(b) and 4(a) we see that the non-diagonal terms affect more severely the higher angular momentum region by decreasing the staggering amplitude. Moreover, the larger values for the parameters of the non-diagonal terms, shown in Fig. 4(b), reduce more seriously the staggering pattern in the higher angular momentum region, as one can see by comparing Figs 3(b) and 4(b) and noticing the different scales on the vertical axes of the two figures. The pattern appearing in Fig. 4(b) resembles the experimental situation in ${ }^{218} \mathrm{Rn}$ and ${ }^{228} \mathrm{Th}$ [5] (odd-even staggering with amplitude decreasing as a function of $I$ ).

\section{Low- $K(K \leq 3)$ analysis of the model formalism}

A discussion on the $K$-values appearing in our model consideration is appropriate in this place.

Usually from microscopic point of view the eigenvalues of the total angular momentum projection $I_{z}$ (which, in case of axial symmetry, coincides with the intrinsic momentum projection $J_{z}$ ) are restricted to $K=\leq 3$. This requirement is well justified in the case of octupole vibrations of quadrupole deformed nuclei. It reflects the shell model concept that the octupole degrees of freedom could be generated by an octupole-octupole $\lambda=3$ interaction (where $\lambda$ stands, as usual, for the multipolarity) which couples single-particle states of opposite parity [1]. In particular, this assumption gives a satisfactory interpretation for the fine structure of negative parity rotational bands built on octupole vibrations.

In view of the above, it is interesting to examine our formalism in the case when the space of the $K$-values is restricted to $K_{\max }=3$. Indeed such a test is important especially for the low angular momentum regions, where the intrinsic structure of octupole degrees of freedom is microscopically well studied.

Along these lines, we performed a schematic calculation including the values $K=0,1$, 2, 3 in the angular momentum region up to $I=10$, as well as another calculation including the values $K=0,1,2,3$ in the angular momentum region up to $I=16$. The yrast sequences obtained are given in Tables 2 and 3 respectively, while the resulting staggering patterns are shown in Fig. 5. We see that our formalism provides a regular staggering pattern for the states up to $I=10$ [Fig. 5(a)] and a "beat" pattern for the spectrum up to $I=16$ [Fig. 5(b)]. These examples (obtained for the parameter values given in Tables 2 and 3, respectively) illustrate that our approach is capable to provide reasonable staggering patterns under the restriction to $K \leq 3$, at least in the low angular momentum region with $I=10-16$. In other words, the schematic model gives reasonable results under the restriction to $K \leq 3$, at least in the microscopically accessible angular momentum regions. 
It is, however, important to remark that our approach suggests a rather more extended treatment of the collective octupole degrees of freedom, which is expected to be useful in view of the increasing bulk of data indicating possible stable octupole deformations in several nuclei, the light actinides providing the best examples. Some of these bands (the octupole bands in ${ }^{224-226} \mathrm{Ra}$ and ${ }^{224-228} \mathrm{Th}$, for example) possess rather well pronounced rotational structures (see Table 1 in Ref. [5]). Our proposition is that in these cases the concept of a rotating quadrupole-octupole shape should be more evident. In these cases, in contrast to the pure octupole vibrations, it is not a priori clear why the combined quadrupole-octupole intrinsic configurations should be restricted to $K \leq 3$ projection values. Namely for these kinds of rotational motion our collective formalism suggests that $K$ could be higher than 3. One can easily see that for the relatively well deformed nuclei, such as ${ }^{224-226} \mathrm{Ra}$ and ${ }^{224-228} \mathrm{Th}$, the Nilsson orbitals do not exclude the presence of reasonable $K>3$ intrinsic configurations [21]. This remark is of particular importance for the higher angular momentum region $(I \geq 10-16)$, where pair breaking effects are possible.

Another important question to be examined is whether the energy gain in case of yrast rotations with $K>3$ is larger than the intrinsic energy necessary to raise $K$ above 3 . There is no a priori answer to this question, which would be an interesting problem for future combined efforts from both collective and microscopic points of view. Here we just remark that for the collective motion considered the energy gain rapidly increases with the increase of the angular momentum (see the depth of the minima in the curves of Fig. 1).

\section{Further tests of the model formalism}

The restriction to $K \leq 3$ values, used in the previous section, demonstrated that odd-even staggering and "beat" patterns can indeed appear in this special case. This particular collection of $K$ values $(K=0,1,2,3)$ has certain probability to appear low in energy in the case of nuclei exhibiting octupole deformation, but other possibilities also exist. In fact, it is well known from experiment that the various $K$ values are built up through the pair-breaking mechanism. As a result, $K$ is not a smoothly increasing function (while $I$ is). Which $K$ values will appear lower in energy in each nucleus depends strongly on the microscopic structure of the nucleus. One is therefore confronted with the following questions:

1) Given a collection of $K$ values, which appear low in energy in a specific nucleus because of microscopic reasons, is the present formalism predicting odd-even staggering or not?

2) What is the meaning of minimization with respect to $K$ in this case?

3) In which order do the various allowed values of $K$ appear as $I$ is increasing?

We have performed several numerical tests, which need not to be reproduced here in detail, in order to answer these questions. The following conclusions have been reached:

1 ) Given a collection of $K$ values which are non-successive integers (for example, $K=5$, $7,9,10,15)$, odd-even staggering and "beat" patterns do appear for certain values of the Hamiltonian parameters. One is therefore persuaded that in principle the model formalism can produce "beat" patterns for any microscopically directed collection of $K$ values.

2) In the just mentioned case, $K$ is not a continuous variable. However, one can still minimize the energy with respect to $K$, in the following sense: Given a collection of $K$ 
values, for each value of $I$ one determines (among the members of the collection) the $K$ value for which the energy is minimum.

3) In all cases the allowed values of $K$ appear in increasing order as $I$ is increasing, similarly to what occurs in Tables $1-3$.

In all of the above we have been assuming that $K$ takes only integer values, i.e. that $K$ is a good quantum number. This is an assumption of the model, which is quite usual in the realm of phenomenological models (but not in the case of microscopic models), in which $K$ is assumed to be a good (or asymptotically good) quantum number. One might wonder, however, how strongly the appearance of odd-even staggering and "beat" patterns in the present model depends on this assumption. In order to confront this question, we have performed the following tests: We repeated the calculations concerning Figs. 3(a) and 3(b) allowing $K$ to vary not with a step of 1.0 , but with a step of 0.5 , or 0.1 , or 0.01 , or 0.0001 , as well as allowing $K$ to be a continuous variable. The results need not be reproduced here in full detail, but the conclusion was that in both cases the odd-even staggering drops dramatically as the step of the variation of $K$ decreases, and practically vanishes when $K$ is a continuous variable. One therefore concludes that the appearence of odd-even staggering and "beat" patterns in the present model strongly depends on the assumption that $K$ is a good (or asymptotically good) quantum number, taking integer values, an assumption which is rather common among phenomenological models. In the other hand, as it has already been mentioned in the beginning of this section, practically any microscopically directed set of integer values of $K$ can lead to the appearance of odd-even staggering and "beat" patterns within the framework of the present model.

In conclusion, the assumption that $K$ is a good (or asymptotically good) quantum number, therefore taking integer values only, is a fundamental one as far as the appearance of odd-even staggering and "beat" patterns within the present model is concerned. Once $K$ is assuming only integer values, odd-even staggering and "beat" patterns can in principle be constructed (for appropriate parameter values in the Hamiltonian) for any set of microscopically directed $K$ values lying low in energy.

\section{Discussion}

The staggering patterns illustrated so far (Figs 3 and 4) cover almost all odd-even staggering patterns seen in nuclear octupole bands. The amplitudes obtained for the sets of parameters considered vary up to $300 \mathrm{keV}$. Staggering patterns with larger values of $\operatorname{Stg}(I)$ can be easily obtained for different parameter sets. On this basis it is reasonable to assume that the model parameters can be adjusted appropriately so as to reproduce the staggering patterns seen in the octupole bands of the light actinides [5], as well as in rotational negative parity bands with $K \geq 1$ built on octupole vibrations. In the fitting procedure one should take into account the minimization of the energy with respect to $K$ for each given value of $I$. Work in this direction is in progress.

At this point the following comments on the structure of the collective interactions used and the related symmetries are in place:

1) The above mentioned fact that the six non-diagonal terms of the octupole Hamiltonian can be arranged into three pairs, the terms belonging to the same pair giving equal contributions, indicates that only four terms of the octupole Hamiltonian (the diagonal 
term plus three appropriately chosen non-diagonal terms) suffice for the determination of the energy spectrum. This result reflects the fact that in the intrinsic frame of reference three octupole degrees of freedom, from the total of seven ones, are related to the orientation angles. In the present case we chose in Sec. 5 and in Fig. 4 to keep the diagonal term $\hat{H}_{F_{1}(1)}$ and the non-diagonal terms $\hat{H}_{F_{1}(2)}, \hat{H}_{F_{2}(1)}$ and $\hat{H}_{F_{2}(2)}$. Our analysis (related to the collective rotations of the system) gives a natural way for determining the four collective octupole interaction terms which give independent contributions.

2) The symmetry of the various combinations of spherical harmonics appearing in Eqs (3)-(9) has been considered in detail in Ref. [10]. The $Y_{30}$ term is axially symmetric, i.e. it has the symmetry of the $\mathrm{D}_{\infty}$ group, while the term $Y_{31}-Y_{3-1}$ has the symmetry of the $\mathrm{C}_{2 v}$ group, the term $Y_{32}+Y_{3-2}$ has the symmetry of the $\mathrm{T}_{d}$ group, and the term $Y_{33}-Y_{3-3}$ has the symmetry of the $\mathrm{D}_{3 h}$ group. ¿From the correspondence between Eqs (3)-(9) and Eqs (11)-(17) we see that the diagonal term $\hat{H}_{F_{1}(1)}$ is axially symmetric, while the non-diagonal terms $\hat{H}_{F_{1}(2)}, \hat{H}_{F_{2}(1)}$ and $\hat{H}_{F_{2}(2)}$ (mentioned in the previous item 1) ) are constructed by using the combinations $\left(Y_{31}-Y_{3-1}\right),\left(Y_{32}+Y_{3-2}\right)$, and $\left(Y_{33}-Y_{3-3}\right)$.

3) Concerning the role of the various terms in the odd-even staggering effect, our schematic results of Secs. 4 and 5 indicate that the non-diagonal $K$-bandmixing interactions suppress the staggering pattern, while the axially symmetric term $\hat{H}_{F_{1}(1)}$ is able to provide a "beat" staggering behavior (see Fig. 3(e)). The quadrupole-octupole term $\hat{H}_{q o c}$ does influence the staggering pattern, providing wider angular momentum regions with regular staggering. In short, the axially symmetric term seems to be the most important one (among the terms involving the octupole degrees of freedom) for the production of "beat" behavior in the odd-even staggering pattern. However, it should be remembered at this point that the octupole and octupole-quadrupole terms are not the only ones contributing to the odd-even staggering effect. As we have already mentioned in Sec. 4, the term $f_{k} K$, coming from the bandhead energy, makes to the odd--even staggering a contribution of constant amplitude, while the $A^{\prime} K^{2}$ term, coming from the quadrupole part of the Hamiltonian, makes a contribution to the odd-even staggering with an amplitude which is increasing linearly as a function of $I$. It should be noticed, though, that these remarks are based only on a few schematic calculations and in no way are final conclusions. In order to reach safer conclusions about the relative importance of the various terms in giving rise to a "beat" behavior of the odd-even staggering, one has to perform detailed fits to the octupole bands of the light actinides, as mentioned above.

4) As has already been commented is Secs. 4 and 6 the most general schematic results we have obtained suggest that in the high angular momentum region some high $K$ values should be involved (as a result of the minimization of the energy with respect to $K$ for each given value of $I$, which is an important ingredient of our approach). The fact that the $K$ quantum number is not a good quantum number (not even approximately) of the relevant states has been realized long ago [22, 23, 24]. In microscopic calculations in the rare earth region (with $152 \leq A \leq 190$ ) [22], in which the values $K=0,1,2,3$ have been included, it has been seen that in the beginning of the region the values $K=0,1$ are important for the lowest $3^{-}$state, while in the middle of the region the values $K=1,2$ are important and in the far end of the region the values $K=2,3$ are important [22]. These results are consistent with our low- $K$ model analysis given in Section 6 . The same authors have dealt with the actinide region $(A \geq 222)$ in Ref. [23]. One of the authors of Refs [22, 23] in Ref. 
24] finds that the restriction to $K \leq 3$ is not justifiable for large energies. These findings are in agreement with our results given in Table 1 as well as with our comments in the end of Sec. 6.

5) Concerning the analysis in Sec. 5 and the above comments 3) and 4), we remark that the restrictions imposed on the non-diagonal Hamiltonian terms (keeping $K$ asymptotically good) are rather strong and reflect the particular physical assumptions of our consideration. However, for a more general quantum mechanical system there is no principal reason to restrict the analysis by assuming the presence of small non-axial deformations only [25]. In this direction an extension of the present work not limited to small non-diagonal contributions could be of interest.

\section{Conclusions and outlook}

In summary, we have considered a Hamiltonian involving octupole and quadrupole terms, along with an octupole-quadrupole interaction and a $K$-dependent bandhead term. The octupole terms have been classified by using the irreducible representations (irreps) of the octahedron group O. By minimizing the energy with respect to the angular momentum projection $K$ for each given value of the angular momentum $I$ we have reached the conclusion that $K$ is increasing with increasing $I$, even in the case in which $K$ is allowed to assume only a resticted set of microscopically dictated values, a result which can be interpreted as corresponding to a wobbling motion. Various terms of the Hamiltonian give rise to odd-even staggering depending on $I$ in different ways, in this way making the Hamiltonian able to produce several different odd-even staggering patterns, some of which have been observed in the octupole bands of light actinides. In order to examine the relative importance of the various terms giving rise to odd-even staggering, detailed fits of the octupole bands of the light actinides should be performed, a procedure which is not very simple because of the above mentioned minimization procedure involved in it. In addition, the applicability of the present formalism to rotational bands with $K \geq 1$ built on octupole vibrations, which also demonstrate odd-even staggering effects, should be examined. The present Hamiltonian, because of the variety of odd-even staggering patterns it can produce, seems to be a good starting point for systematizing the different odd-even staggering patterns seen in octupole bands, as well as in rotational bands built on octupole vibrations. Work in this direction is in progress.

\section{Acknowledgments}

The authors are grateful to Prof. P. Quentin for several illuminating discussions at various stages of the development of this work, as well as to Prof. S. Pittel for a careful reading of manuscript and useful comments. One of authors (NM) is grateful to Prof. N. Lo Iudice for hospitality during his stay in Università di Napoli "Federico II" and for detailed discussions on the subject of this work. This work has been supported by the Bulgarian National Fund for Scientific Research under contract no MU-F-02/98. Last but not least, the authors are grateful to an unknown referee, whose questions and suggestions led to the development of Sections 6 and 7 of the article.

This article is dedicated to the memory of Roussy Petkov Roussev, dearest colleague and friend, who passed away soon after the compilation of this work. 


\section{Appendix}

Non-zero matrix elements of the operators of Eqs (11)-(17) in the states $|I K\rangle$ with total angular momentum $I$ and projection $K$, with $X=I(I+1)$ :

$$
\begin{aligned}
\left\langle I K+2\left|\hat{H}_{A_{2}}\right| I K\right\rangle & =\frac{i}{4} a_{2}(K+1) \sqrt{X-(K+1)(K+2)} \sqrt{X-K(K+1)}, \\
\left\langle I K-2\left|\hat{H}_{A_{2}}\right| I K\right\rangle & =-\frac{i}{4} a_{2}(K-1) \sqrt{X-(K-1)(K-2)} \sqrt{X-K(K-1)}, \\
\left\langle I K\left|\hat{H}_{F_{1}(1)}\right| I K\right\rangle & =\frac{1}{2} f_{11} K\left(5 K^{2}-3 X\right), \\
\left\langle I K+1\left|\hat{H}_{F_{1}(2)}\right| I K\right\rangle & =\frac{1}{16} f_{12}\left(3 X-15 K^{2}-15 K-10\right) \sqrt{X-K(K+1)}, \\
\left\langle I K-1\left|\hat{H}_{F_{1}(2)}\right| I K\right\rangle & =\frac{1}{16} f_{12}\left(3 X-15 K^{2}+15 K-10\right) \sqrt{X-K(K-1)}, \\
\left\langle I K+1\left|\hat{H}_{F_{1}(3)} I\right| K\right\rangle & =\frac{i}{16} f_{13}\left(3 X-15 K^{2}-15 K-10\right) \sqrt{X-K(K+1)}, \\
\left\langle I K-1\left|\hat{H}_{F_{1}(3)}\right| I K\right\rangle & =-\frac{i}{16} f_{13}\left(3 X-15 K^{2}+15 K-10\right) \sqrt{X-K(K-1)} \\
\left\langle I K+2\left|\hat{H}_{F_{2}(1)}\right| I K\right\rangle & =\frac{1}{2} f_{21}(K+1) \sqrt{X-(K+1)(K+2)} \sqrt{X-K(K+1)} \\
\left\langle I K-2\left|\hat{H}_{F_{2}(1)}\right| I K\right\rangle & =\frac{1}{2} f_{21}(K-1) \sqrt{X-(K-1)(K-2)} \sqrt{X-K(K-1)} \\
\left\langle I K+1\left|\hat{H}_{F_{2}(2)}\right| I K\right\rangle & =\frac{1}{8} f_{22}\left(X-5 K^{2}-5 K-2\right) \sqrt{X-K(K+1)}, \\
\left\langle I K-1\left|\hat{H}_{F_{2}(2)}\right| I K\right\rangle & =\frac{1}{8} f_{22}\left(X-5 K^{2}+5 K-2\right) \sqrt{X-K(K-1)}, \\
\left\langle I K+1\left|\hat{H}_{F_{2}(3)}\right| I K\right\rangle & =-\frac{i}{8} f_{23}\left(X-5 K^{2}-5 K-2\right) \sqrt{X-K(K+1)}, \\
\left\langle I K-1\left|\hat{H}_{F_{2}(3)}\right| I K\right\rangle & =\frac{i}{8} f_{23}\left(X-5 K^{2}+5 K-2\right) \sqrt{X-K(K-1)} .
\end{aligned}
$$




\section{References}

[1] A. Bohr and B. R. Mottelson, Nuclear Stucture vol. II (Benjamin, New York, 1975).

[2] S. G. Rohoziński, Rep. Prog. Phys. 51, 541 (1988).

[3] I. Ahmad and P. A. Butler, Annu. Rev. Nucl. Part. Sci. 43, 71 (1993).

[4] P. A. Butler and W. Nazarewicz, Rev. Mod. Phys. 68, 349 (1996).

[5] D. Bonatsos, C. Daskaloyannis, S. B. Drenska, N. Karoussos, N. Minkov, P. P. Raychev and R. P. Roussev, Phys. Rev. C 62, 024301 (2000).

[6] J. F. C. Cocks, P. A. Butler, K. J. Cann, P. T. Greenlees, G. D. Jones, S. Asztalos, P. Bhattacharyya, R. Broda, R. M. Clark, M. A. Deleplanque, R. M. Diamond, P. Fallon, B. Fornal, P. M. Jones, R. Julin, T. Lauritsen, I. Y. Lee, A. O. Macchiavelli, R. W. MacLeod, J. F. Smith, F. S. Stephens and C. T. Zhang, Phys. Rev. Lett. 78, 2920 (1997).

[7] J. F. C. Cocks, D. Hawcroft, N. Amzal, P. A. Butler, K. J. Cann, P. T. Greenlees, G. D. Jones, S. Asztalos, R. M. Clark, M. A. Deleplanque, R. M. Diamond, P. Fallon, I. Y. Lee, A. O. Macchiavelli, R. W. MacLeod, F. S. Stephens, P. Jones, R. Julin, R. Broda, B. Fornal, J. F. Smith, T. Lauritsen, P. Bhattacharyya and C. T. Zhang, Nucl. Phys. A 645, 61 (1999).

[8] S. G. Rohoziński, J. Phys. G 16, L173 (1990).

[9] I. Hamamoto, X. Z. Zhang and H. Xie, Phys. Lett. B 257, 1 (1991).

[10] I. Hamamoto, B. Mottelson, H. Xie and X. Z. Zhang, Z. Phys. D 21, 163 (1991).

[11] C. Wexler, G. G. Dussel, Phys. Rev. C 60, 014305 (1999).

[12] S. G. Rohoziński, M. Gajda and W. Greiner, J. Phys. G 8, 787 (1982).

[13] W. D. Luo and X. J. Li, Chin. Phys. Lett. 16, 342 (1999).

[14] W. Greiner and J. A. Maruhn, Nuclear Models (Springer-Verlag, Berlin, 1996).

[15] I. Hamamoto and B. Mottelson, Phys. Lett. B 333, 294 (1994).

[16] I. N. Mikhailov and P. Quentin, Phys. Rev. Lett. 74, 3336 (1995).

[17] A. Ansari, M. Oi, N. Onishi, and T. Horibata, Phys. Lett. B 460, 24 (1999).

[18] S. Flibotte, H. R. Andrews, G. C. Ball, C. W. Beausang, F. A. Beck, G. Belier, T. Byrski, D. Curien, P. J. Dagnall, G. de France, D. Disdier, G. Duchêne, Ch. Finck, B. Haas, G. Hackman, D. S. Haslip, V. P. Janzen, B. Kharraja, J. C. Lisle, J. C. Merdinger, S. M. Mullins, W. Nazarewicz, D. C. Radford, V. Rauch, H. Savajols, J. Styczen, Ch. Theisen, P. J. Twin, J. P. Vivien, J. C. Waddington, D. Ward, K. Zuber and S. Åberg, Phys. Rev. Lett. 71, 4299 (1993). 
[19] S. Flibotte, G. Hackman, I. Ragnarsson, Ch. Theisen, H. R. Andrews, G. C. Ball, C. W. Beausang, F. A. Beck, G. Bélier, M. A. Bentley, T. Byrski, D. Curien, G. de France, D. Disdier, G. Duchêne, B. Haas, D. S. Haslip, V. P. Janzen, P. M. Jones, B. Kharraja, J. A. Kuehner, J. C. Lisle, J. C. Merdinger, S. M. Mullins, E. S. Paul, D. Prévost, D. C. Radford, V. Rauch, J. F. Smith, J. Styczen, P. J. Twin, J. P. Vivien, J. C. Waddington, D. Ward and K. Zuber, Nucl. Phys. A 584, 373 (1995).

[20] B. Cederwall, R. V. F. Janssens, M. J. Brinkman, I. Y. Lee, I. Ahmad, J. A. Becker, M. P. Carpenter, B. Crowell, M. A. Deleplanque, R. M. Diamond, J. E. Draper, C. Duyar, P. Fallon, L. P. Farris, E. A. Henry, R. G. Henry, J. R. Hughes, T. L. Khoo, T. Lauritsen, A. O. Macchiavelli, E. Rubel, F. S. Stephens, M. A. Stoyer, W. Satuła, I. Wiedenhoever and R. Wyss, Phys. Rev. Lett. 72, 3150 (1994).

[21] P. Quentin, private communication (2000).

[22] K. Neergård and P. Vogel, Nucl. Phys. A 145, 33 (1970).

[23] K. Neergård and P. Vogel, Nucl. Phys. A 149, 217 (1970).

[24] P. Vogel, Phys. Lett. B 60, 431 (1976).

[25] S. Pittel, private communication (2000). 
Table 1: The "yrast" energy levels, $E(I)$ (in keV), and the respective $K$-values (in $\hbar$ ) obtained from Eq. (25) for the parameter set $E_{0}=500 \mathrm{keV}, f_{k}=-7.5 \mathrm{keV}, A=12 \mathrm{keV}$, $A^{\prime}=6.6 \mathrm{keV}, f_{11}=0.56 \mathrm{keV}, f_{\text {qoc }}=0.085 \mathrm{keV}$, by minimizing the energy with respect to $K$ for each given value of $I$. See Sec. 4 for further discussion.

\begin{tabular}{ccccccccc}
\hline \hline$I$ & $E(I)$ & $K$ & $I$ & $E(I)$ & $K$ & $I$ & $E(I)$ & $\mathrm{K}$ \\
\hline 1 & 522.772 & 1 & 13 & 2335.81 & 5 & 25 & 5453.12 & 11 \\
2 & 568.327 & 1 & 14 & 2576.57 & 6 & 26 & 5694.49 & 12 \\
3 & 637.095 & 1 & 15 & 2827.57 & 6 & 27 & 5935.50 & 12 \\
4 & 728.710 & 1 & 16 & 3082.36 & 7 & 28 & 6157.50 & 13 \\
5 & 840.857 & 2 & 17 & 3344.94 & 7 & 29 & 6378.29 & 13 \\
6 & 971.155 & 2 & 18 & 3608.18 & 8 & 30 & 6575.37 & 14 \\
7 & 1123.22 & 2 & 19 & 3877.05 & 8 & 31 & 6770.62 & 14 \\
8 & 1288.09 & 3 & 20 & 4143.16 & 9 & 32 & 6937.23 & 15 \\
9 & 1472.71 & 3 & 21 & 4413.03 & 9 & 33 & 7101.62 & 15 \\
10 & 1668.56 & 4 & 22 & 4676.45 & 10 & 34 & 7232.21 & 16 \\
11 & 1880.56 & 4 & 23 & 4942.01 & 10 & 35 & 7360.44 & 16 \\
12 & 2101.68 & 5 & 24 & 5197.18 & 11 & 36 & 7449.45 & 17 \\
\hline \hline
\end{tabular}


Table 2: Same as Table 1, but for the parameter set $E_{0}=0 \mathrm{keV}, f_{k}=0 \mathrm{keV}, A=12 \mathrm{keV}$, $A^{\prime}=9.96 \mathrm{keV}, f_{11}=0.75 \mathrm{keV}, f_{q o c}=0.15 \mathrm{keV}$. The restriction $K \leq 3$ has been imposed. See Sec. 6 for further discussion.

\begin{tabular}{cccccc}
\hline \hline$I$ & $E(I)$ & $K$ & $I$ & $E(I)$ & $K$ \\
\hline 1 & 24.00 & 0 & 6 & 487.94 & 1 \\
2 & 72.00 & 0 & 7 & 640.19 & 2 \\
3 & 144.00 & 0 & 8 & 811.21 & 2 \\
4 & 237.97 & 1 & 9 & 994.40 & 3 \\
5 & 351.64 & 1 & 10 & 1194.18 & 3 \\
\hline \hline
\end{tabular}

Table 3: Same as Table 1, but for the parameter set $E_{0}=100 \mathrm{keV}, f_{k}=0.896 \mathrm{keV}$, $A=19.2 \mathrm{keV}, A^{\prime}=18.24 \mathrm{keV}, f_{11}=0.49 \mathrm{keV}, f_{q o c}=0.074 \mathrm{keV}$. The restriction $K \leq 3$ has been imposed. See Sec. 6 for further discussion.

\begin{tabular}{cccccc}
\hline \hline$I$ & $E(I)$ & $K$ & $I$ & $E(I)$ & $K$ \\
\hline 1 & 138.40 & 0 & 9 & 1803.42 & 1 \\
2 & 215.20 & 0 & 10 & 2177.43 & 1 \\
3 & 330.40 & 0 & 11 & 2580.53 & 2 \\
4 & 484.00 & 0 & 12 & 3017.25 & 2 \\
5 & 676.00 & 0 & 13 & 3490.32 & 2 \\
6 & 905.66 & 1 & 14 & 3991.13 & 3 \\
7 & 1167.54 & 1 & 15 & 4521.73 & 3 \\
8 & 1466.79 & 1 & 16 & 5087.69 & 3 \\
\hline \hline
\end{tabular}




\section{Figure Captions}

Figure 1. The diagonal energy matrix element $E_{K}(I)$ (in MeV), Eq. (25), is plotted as a function of $K$ (in $\hbar$ ) for $I=1,2, \ldots, 10$, for the parameter set $E_{0}=500 \mathrm{keV}, f_{k}=-7.5$ $\mathrm{keV}, A=12 \mathrm{keV}, A^{\prime}=6.6 \mathrm{keV}, f_{11}=0.56 \mathrm{keV}, f_{\text {qoc }}=0.085 \mathrm{keV}$.

Figure 2. The diagonal energy matrix element $E_{K}(I)$ (in $\mathrm{MeV}$ ), Eq. (25), is plotted as a function of $I$ (in $\hbar$ ) for $K=10,11,12,13$, for the parameter set of Figure 1.

Figure 3. Odd-even staggering patterns (calculated using Eq. (26) ) as functions of $I$, obtained from the diagonal Hamiltonian of Eq. (24) for several different sets of model parameters, given on the figures. Part (b) corresponds to the same set of parameters as Table 1 and Figs 1 and 2. The first four parameters remain the same in all parts of the figure, except (f).

Figure 4. Odd-even staggering patterns (calculated using Eq. (26)) obtained by adding the three non-diagonal terms $\hat{H}_{F_{1}(2)}$ (Eq. (13)), $\hat{H}_{F_{2}(1)}$ (Eq. (15)), and $\hat{H}_{F_{2}(2)}$ (Eq. (16)) to the diagonal Hamiltonian of Eq. (24), for two different sets of model parameters, given on the figures. The first six parameters are the same as in Figs 1, 2 and 3(b).

Figure 5. Same as Fig. 3, but with the restriction $K \leq 3$ imposed in the angular momentum regions: (a) $I \leq 10$ and (b) $I \leq 16$. The parameter sets correspond to Tables 2 and 3 respectively. 
Figure 1

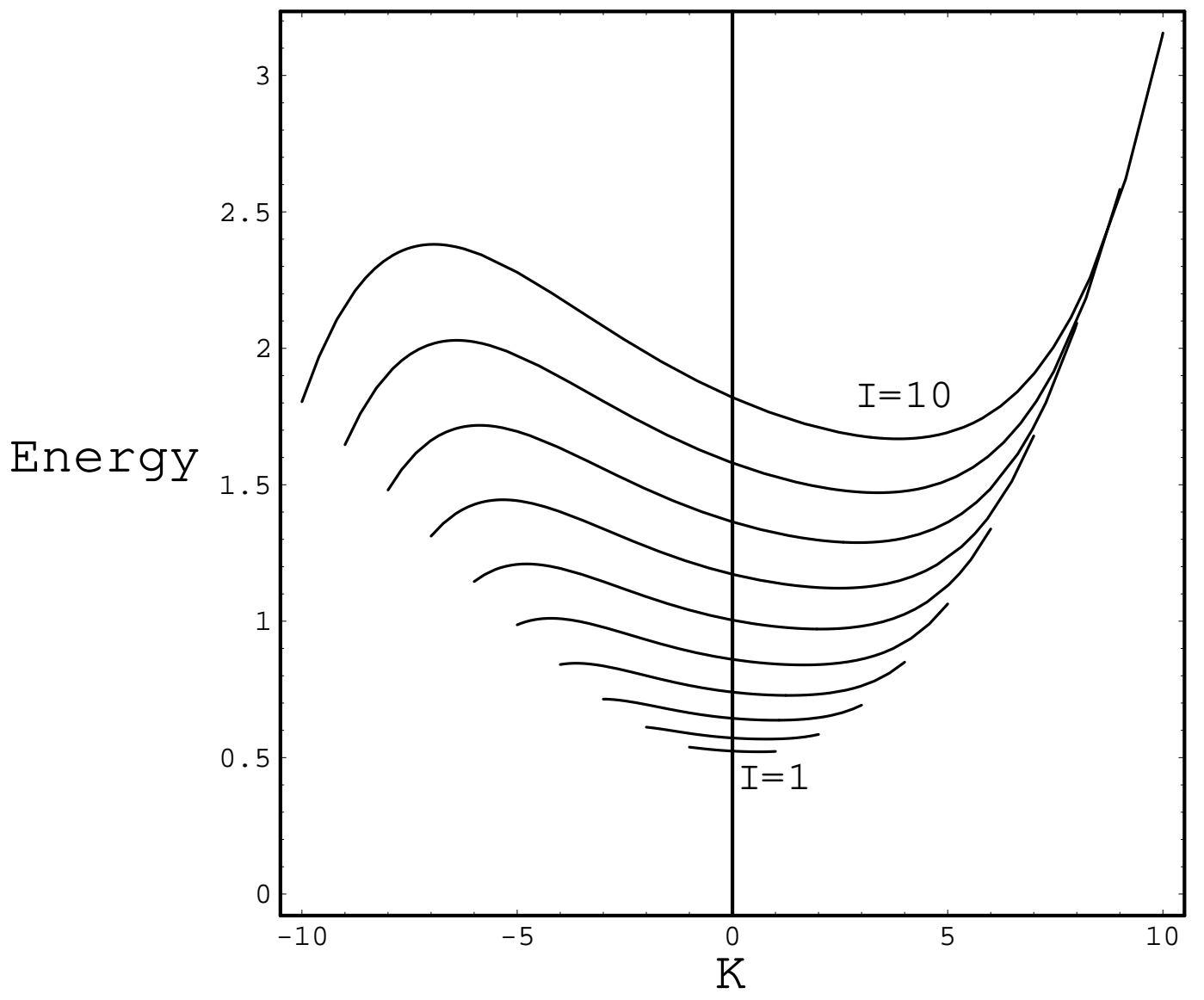


Figure 2

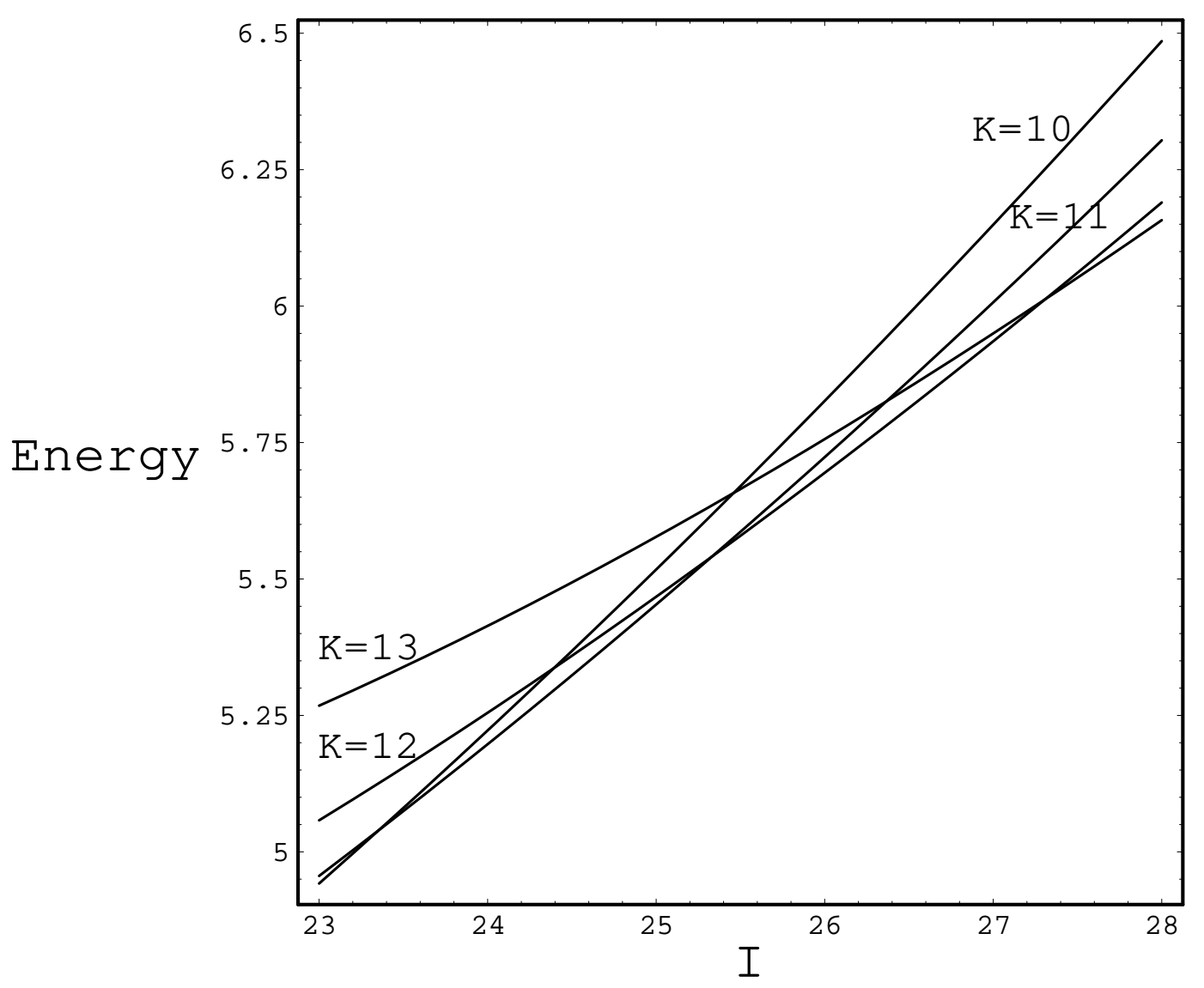


Figure 3(a)

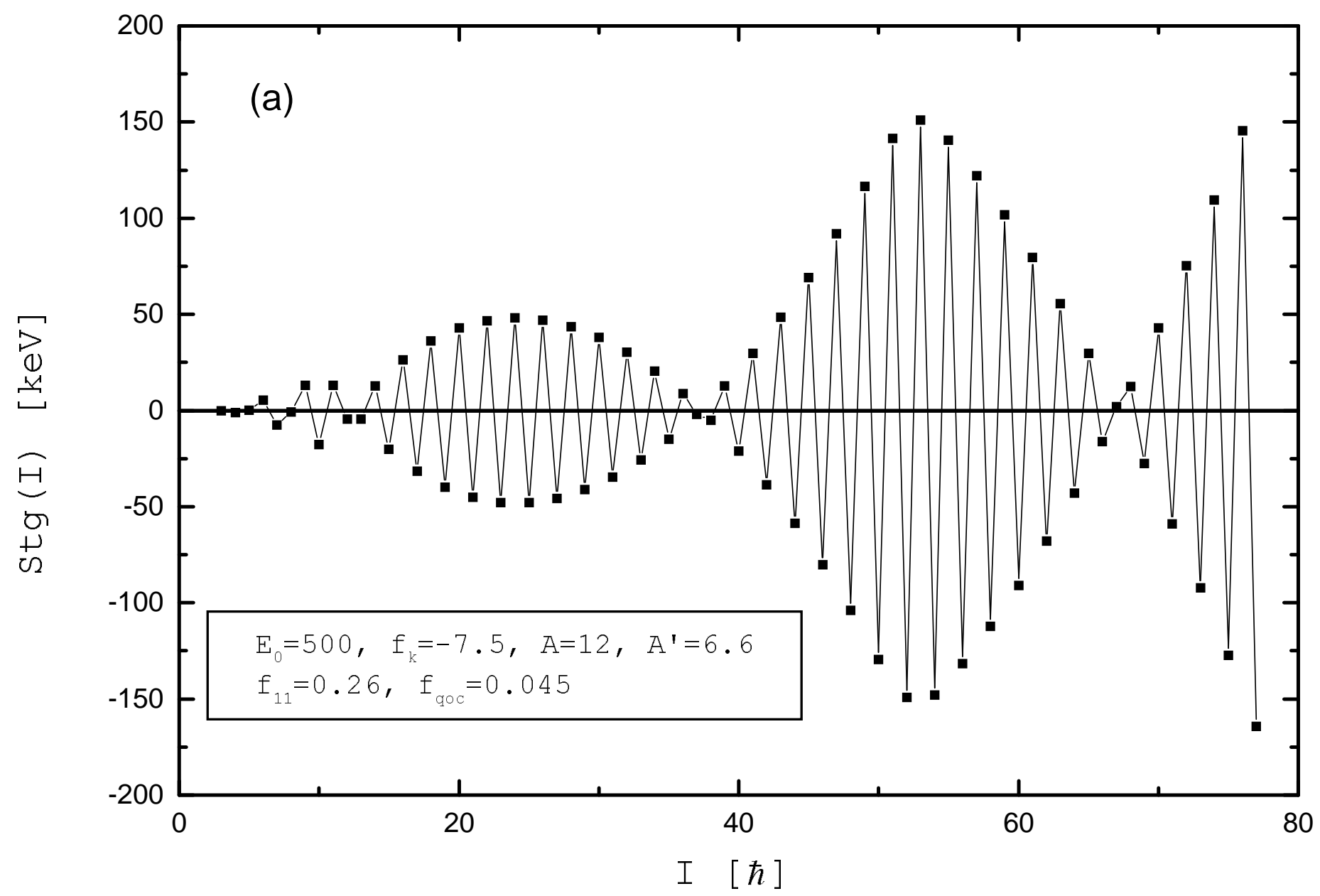


Figure 3(b)

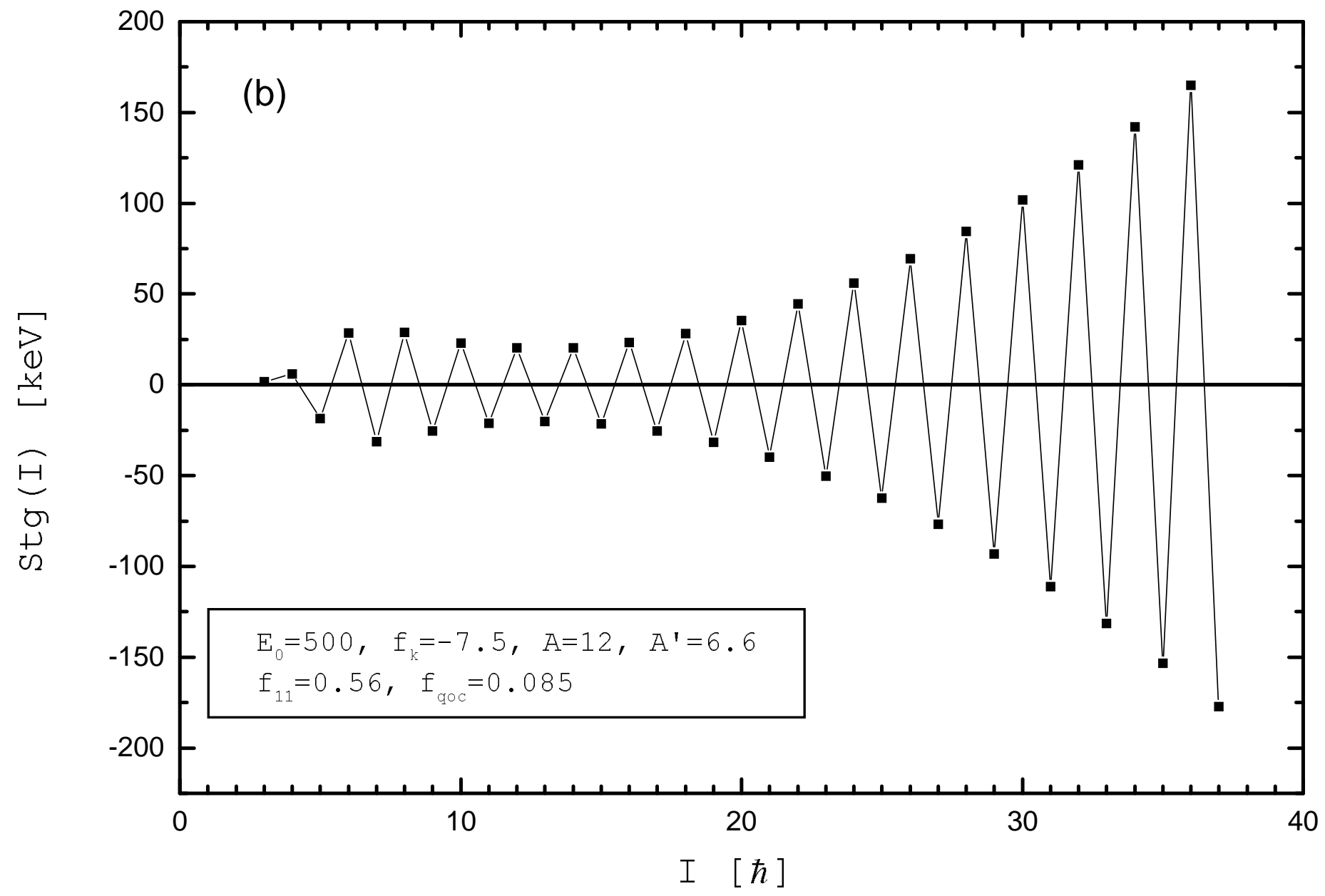


Figure 3(c)

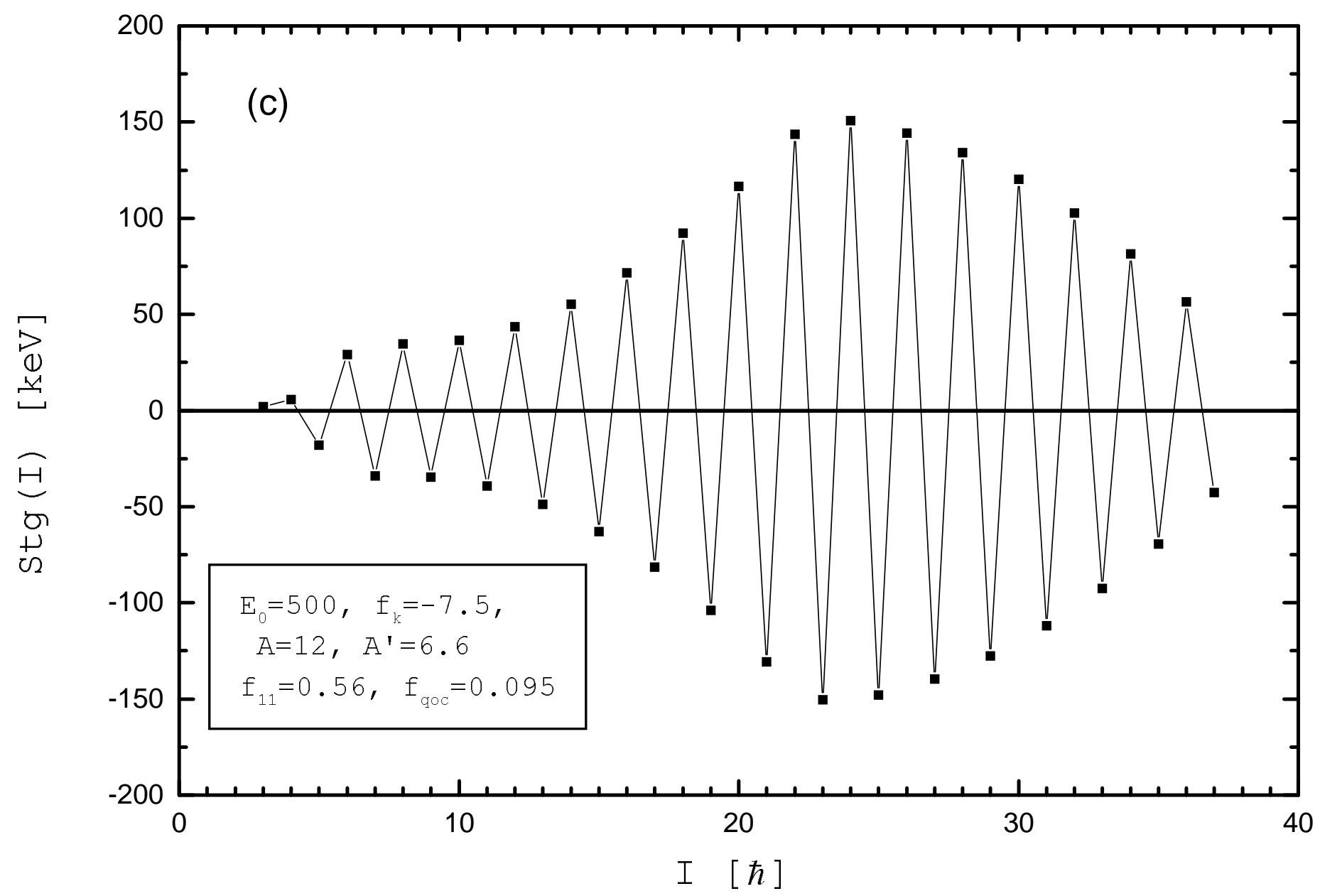


Figure 3(d)

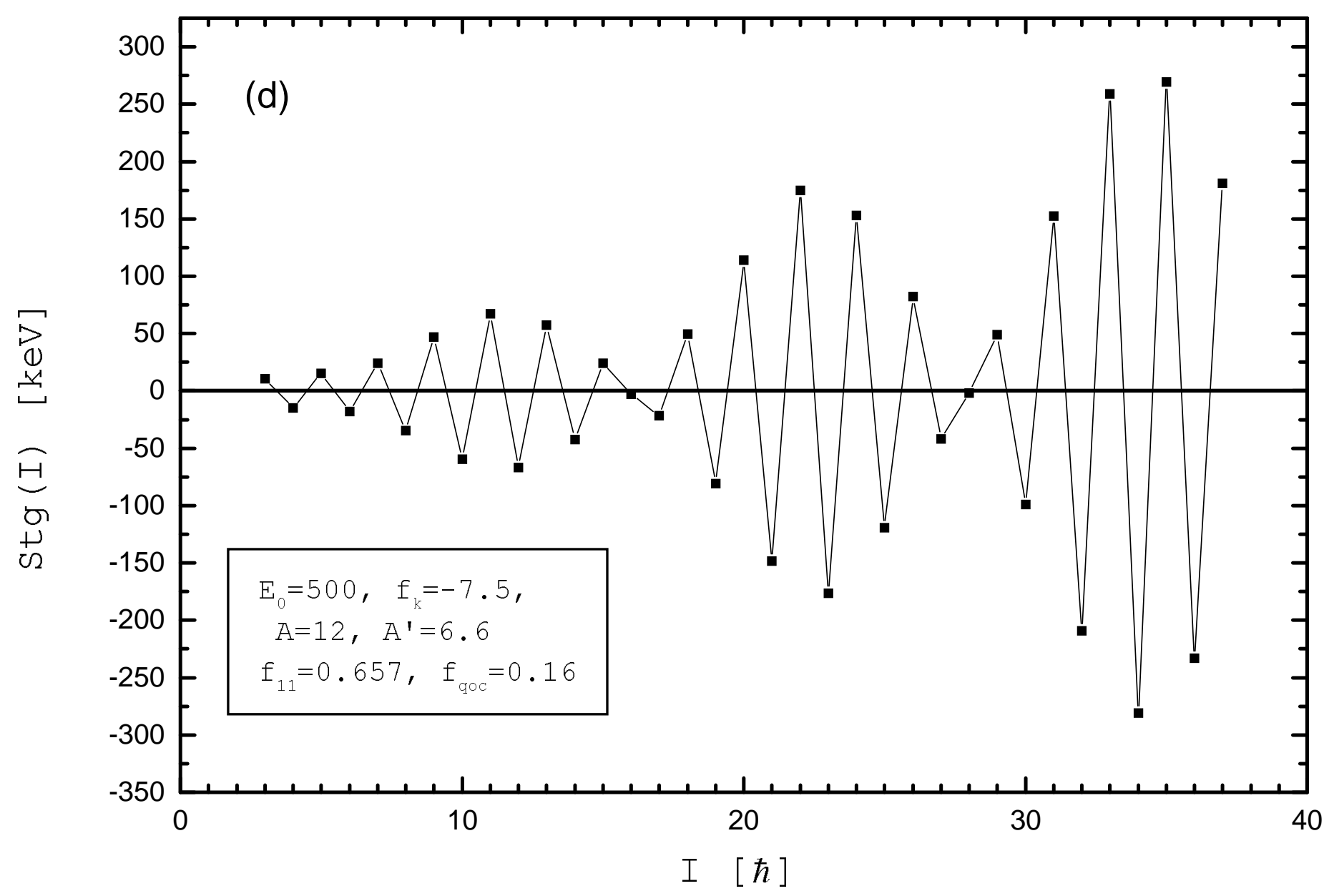


Figure 3(e)

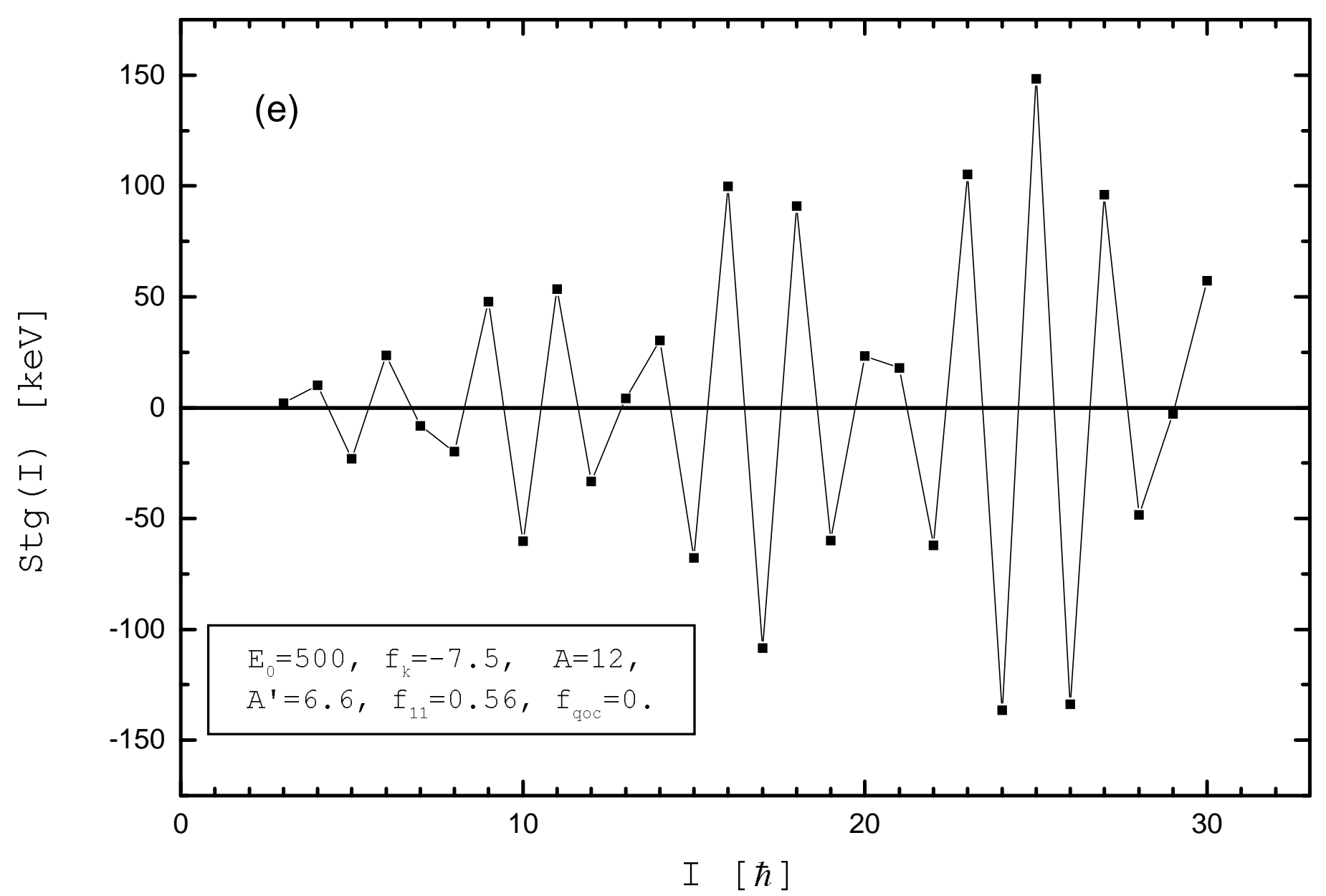


Figure 3(f)

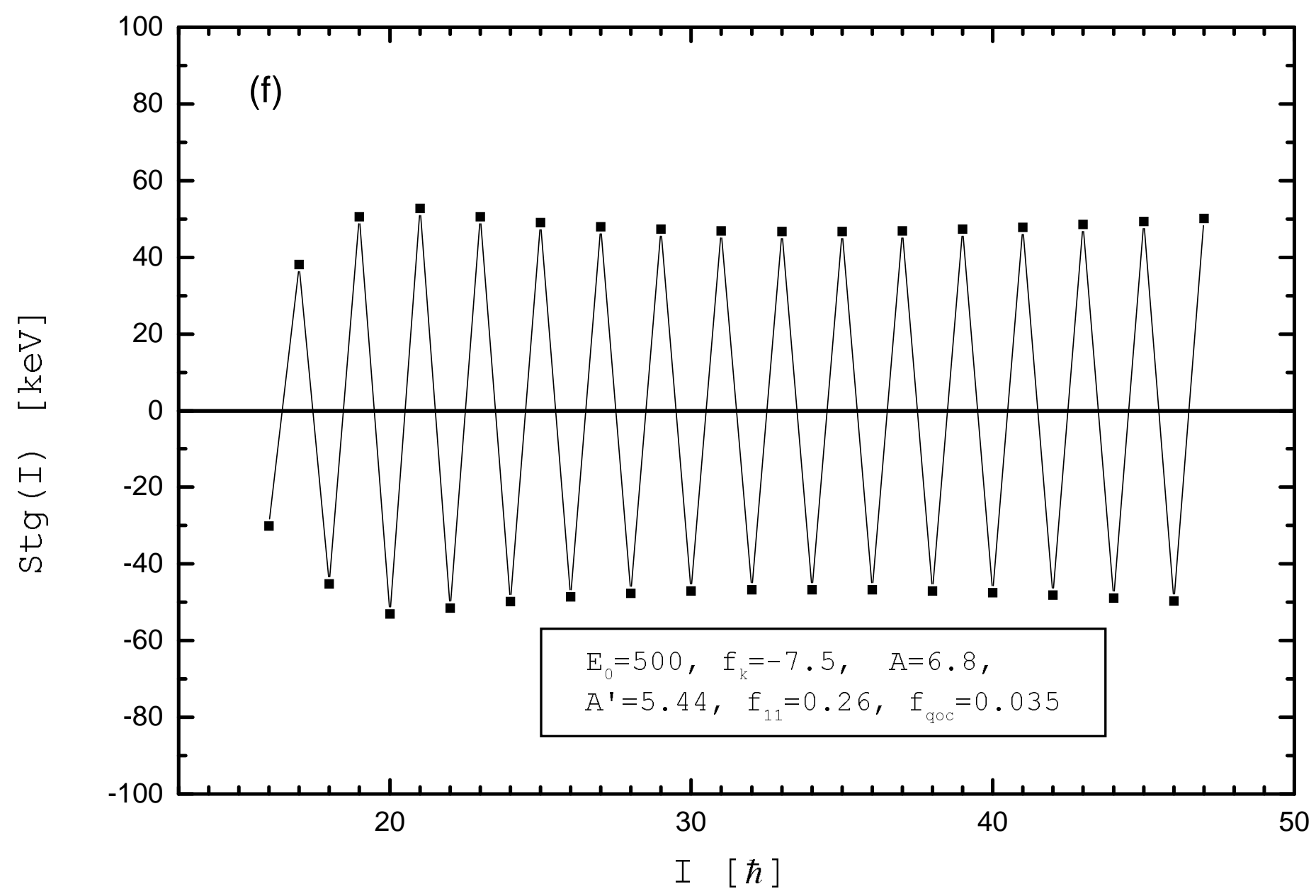


Figure 4(a)

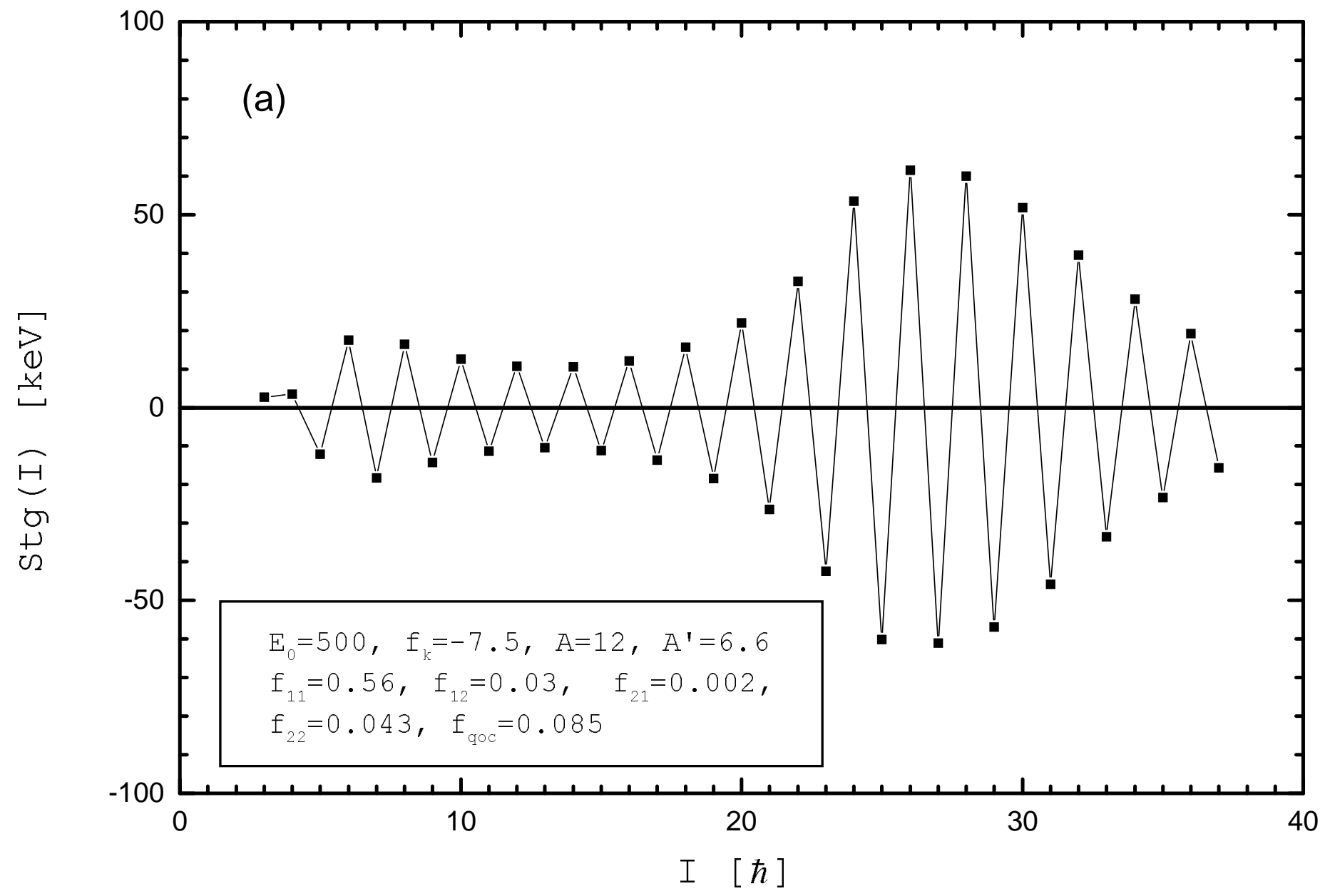


Figure 4(b)

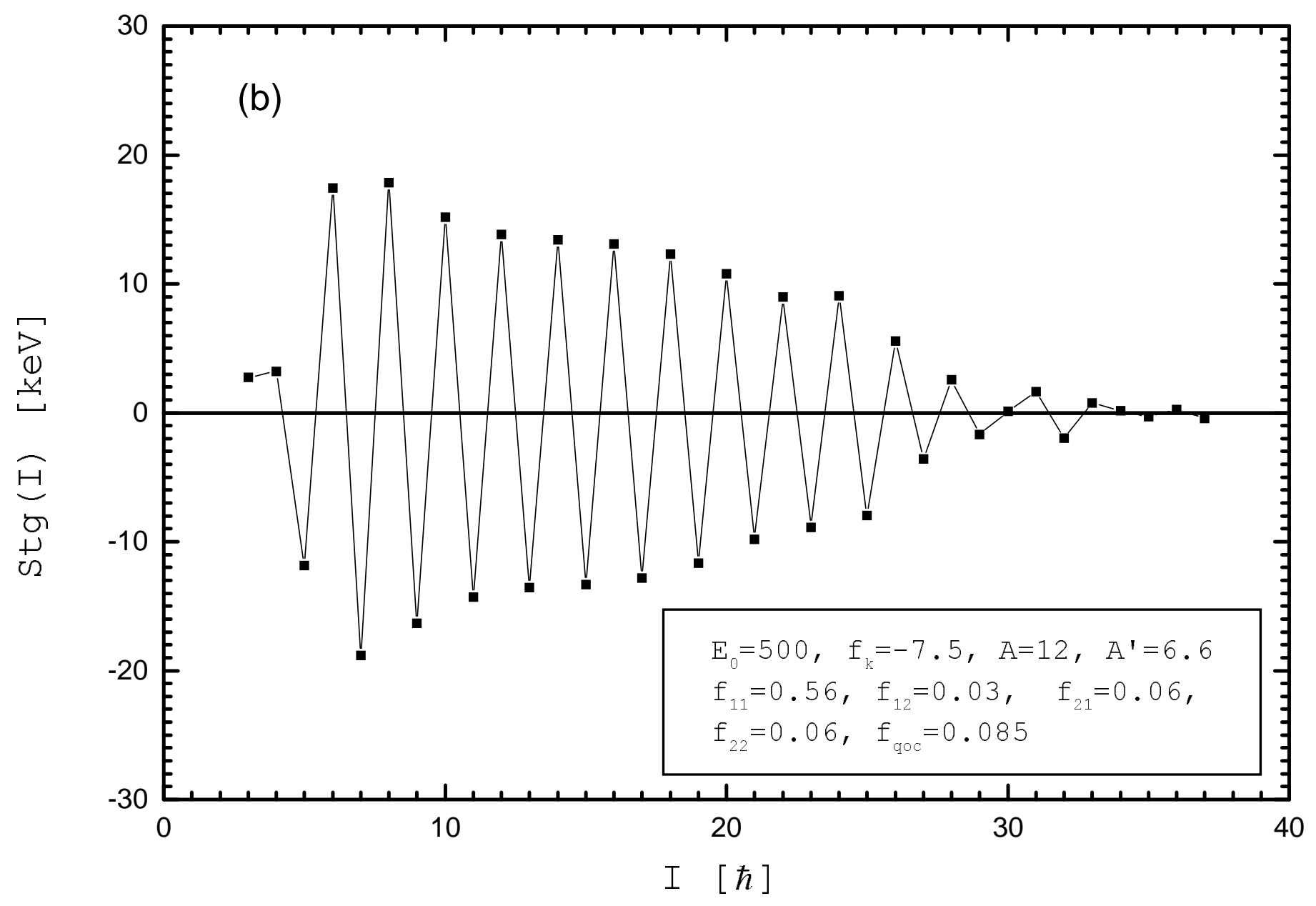


Figure 5(a)

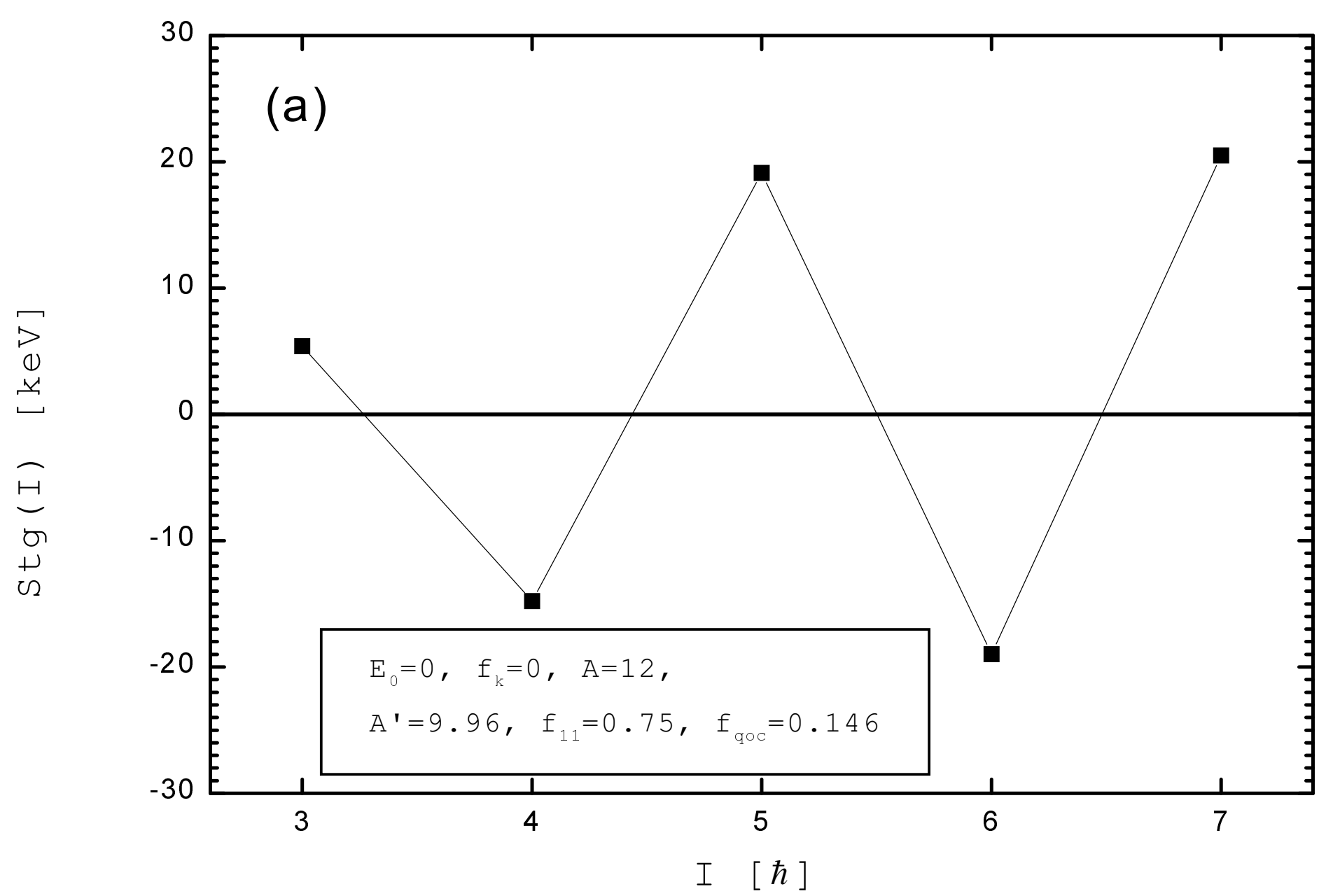


Figure 5(b)

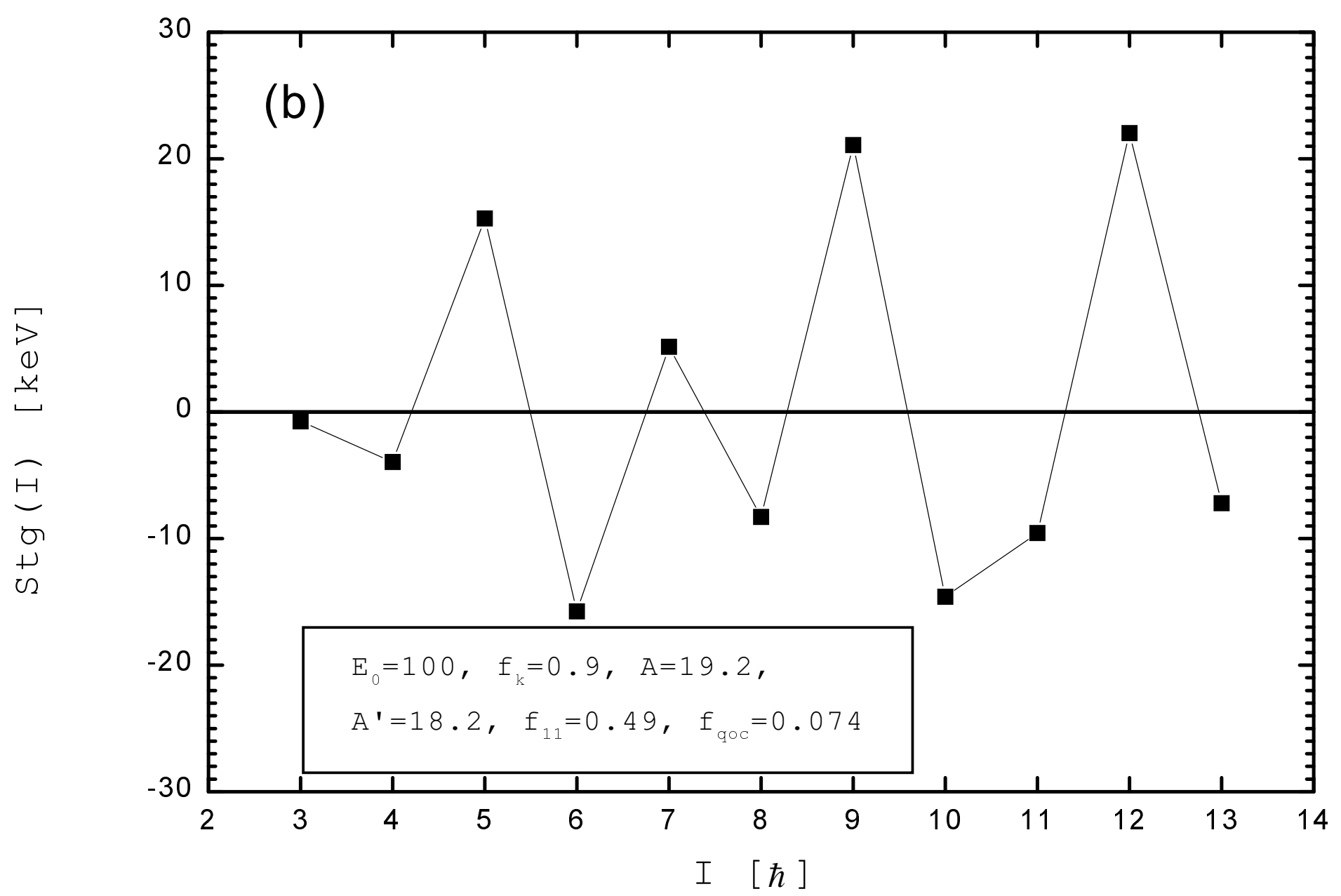

